\title{
CDK4/6-Inhibitor: Lichtblick bei resistentem Mammakarzinom
}

In der Phase-III-Studie PALOMA3

wurde die Wirksamkeit von Palbo-

ciclib in Kombination mit Fulvestrant

bei Patientinnen mit therapierefraktärem, hormonrezeptorpositivem, metastasiertem Brustkrebs bestätigt.

$\mathrm{D}$ as Hormonrezeptor(HR)-positive Mammakarzinom ist abhängig von den zyklinabhängigen Kinasen (CDK) 4 und 6, die den Übergang von der G1- in die S-Phase des Zellzyklus fördern. Dies gilt auch für Brusttumoren, die scheinbar resistent gegenüber der endokrinen Therapie sind. Palbociclib ist ein oraler Inhibitor der CDK 4 und 6. Er zeigte in vitro eine hohe Aktivität und eine synergistische Wirkung in Kombination mit endokrinen Therapien.

In der Phase-III-Studie PALOMA3 wurden Wirksamkeit und Sicherheit von Palbociclib und Fulvestrant bei 521 präund postmenopausalen Frauen mit fortgeschrittenem, EGFR (epidermaler Wachstumsfaktorrezeptor)-2-negativem, HR-positivem Brustkrebs untersucht, bei denen es während der vorangegangenen endokrinen Therapie zu Rezidiv oder Progression gekommen war. Die Patientinnen erhielten randomisiert Palbociclib plus Fulvestrant oder Placebo plus Fulvestrant. Prä- und perimenopausale Frauen bekamen zusätzlich Goserelin.

Nach 195 Fällen von Krankheitsprogression oder Tod erfolgte eine Zwischenanalyse. Der primäre Endpunkt (PFS, progressionsfreies Überleben) wurde bis zu diesem Zeitpunkt erreicht. Das mediane PFS betrug 9,2 Monate in der Gruppe mit Palbociclib und 3,8 Monate in der Placebogruppe (Hazard Ratio für Krankheitsprogression oder Tod 0,42; $\mathrm{p}$ $<0,0001)$. Subgruppenanalysen zeigten, dass der relative Unterschied im PFS bei prä-/perimenopausalen und postmenopausalen Frauen vergleichbar war. 34\% der Patientinnen hatten unter der Kombinationstherapie einen klinischen Nutzen (Ansprechen oder anhaltende stabile Erkrankung), 19\% unter Placebo.

Die häufigsten unerwünschten Ereignisse vom Grad 3/4 in der PalbociclibGruppe waren Neutropenie (62,0 vs.
0,6\%), Leukopenie (25,2 vs. 0,6\%), Anämie (2,6 vs. $1,7 \%)$, Thrombozytopenie (2,3 vs. $0 \%)$ und Fatigue (2,0 vs. 1,2\%). Febrile Neutropenien wurden in beiden Gruppen bei jeweils 0,6\% der Patientinnen beobachtet. Schwerwiegende unerwünschte Ereignisse traten unter der Palbociclib-Kombination seltener auf (9,6 vs. 14,0\%). Die Lebensqualität blieb unter der Kombinationstherapie im Allgemeinen erhalten, verschlechterte sich aber in der Placebogruppe signifikant.

Fazit: Bei Patientinnen mit HR-positivem, metastasiertem Mammakarzinom und Krankheitsprogression unter der endokrinen Therapie führte Palbociclib in Kombination mit Fulvestrant zu einem längeren PFS als die Therapie mit Fulvestrant alleine. Judith Neumaier

Turner NC et al. Palbociclib in Hormone-Receptor-Positive Advanced Breast Cancer. N Engl J Med. 2015;373(3):209-19

\section{Kommentar}

In dieser Interimsanalyse der Studie PALOMA 3 zeigte sich eine relevante Verlängerung des PFS durch die Kombination von Fulvestrant plus Palbociclib im Vergleich zu Fulvestrant plus Placebo. Die Studienteilnehmerinnen litten an einem metastasierten Mammakarzinom und hatten mindestens eine endokrine Vorbehandlung und maximal eine Chemotherapie in der metastasierten Situation erhalten. CDK4/6 spielt eine relevante Rolle in der Regulation der Zellproliferation beim HR-positiven, metastasierten Mammakarzinom - auch bei Progress und damit einer Resistenz unter der klassischen antihormonellen Therapie.

Das Konzept, eine endokrine Therapie mit der zielgerichteten intrazellulären Resistenzüberwindung am CDK4/6-Signal zu kombinieren, kam bisher in allen Paloma-Studien zur Anwendung. In PALOMA 1 wurde Palbociclib in der Erstlinie zusätzlich zu Aromatasehemmern verabreicht [Finn RS et al. AACR. 2014; Abstr CT101]. Interessanterweise wurde Paloma-3 bereits früh für post- und prämenopausale Patientinnen geöffnet (prämenopausal in der Kombination mit GnRH-Analoga) - in beiden Gruppen mit vergleichbar guten Ergebnissen. Auch Patientinnen mit viszeralen Metastasen profitieren von der Palbociclib-Addition. Es gibt in PALOMA 3 keine Patientinnengruppe ohne Nutzen von dieser Therapie. Ein Biomarker zur Patientinnenselektion existiert leider (noch) nicht. Die hohe Lebensqualität in der Verumgruppe ist vor allem auf die Wirkung der Substanz und das aus Patientensicht niedrige Nebenwirkungsspektrum zurückzuführen. Eine relevante Nebenwirkung ist die Hämatotoxizität, mit einer nur geringen Rate an febrilen Neutropenien, die in beiden Studienarmen gleich niedrig war. Dies zeigt wiederum, dass Patientinnen mit neuen

\section{„Alle Patientinnengruppen in PALOMA 3 profitierten von der Palbociclib-Addition."}

oralen Kombinationstherapien noch immer engmaschiger Aufklärung, Überwachung und Betreuung bedürfen. Damit ist die kontinuierliche onkologische Betreuung aus einer Hand weiterhin sinnvoll. Auch wenn die Substanz in den USA bereits zugelassen ist, wird es wichtig sein, die Folgeanalysen - besonders auch bezüglich des Gesamtüberlebens - sowie die Ergebnisse der PALOMA-2-Studie mit Palbociclib in der Erstlinientherapie abzuwarten.

Die Zulassung von Palbociclib in Europa wird für 2016 erwartet. Dann wird Palbociclib auch in Deutschland in das Spektrum der endokrinen und zielgerichteten Therapie beim metastasierten Mammakarzinom aufgenommen werden können. Die Teilnahme deutscher Zentren an den Studienprogrammen beim HR-positiven metastasierten Mammakarzinom erweitert bereits jetzt für Studienpatientinnen die Therapieoptionen, und das Angebot besonders in der näheren Umgebung sollte in jeder Tumorkonferenz und bei jedem Progress für die einzelne Patientin überprüft werden.

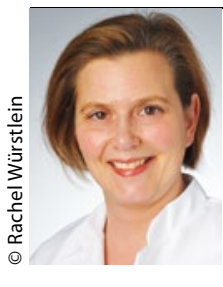

Dr. med. Rachel Würstlein Klinik und Poliklinik für Frauenheilkunde und Geburtshilfe - Universitätsklinikum, München rachel.wuerstlein@med. uni-muenchen.de 